\title{
Verbal ability modulates the associative neighbors effect
}

\author{
InBAL RoN-KAPLAN AND AVISHAI HENIK \\ Ben-Gurion University of the Negev, Beer-Sheva, Israel
}

\begin{abstract}
The number of associative neighbors (ANs) of a word has been found to modulate the associative priming effect (Cañas \& Bajo, 1994). Pilot studies have shown that this influence might be dependent on verbal ability. This possibility was examined by comparing the influence of a prime's ANs on the priming effect in students with high and low verbal ability, using a lexical decision task. Participants with high verbal ability showed a priming effect regardless of ANs. In contrast, low-verbal-ability students showed a significant influence of ANs upon the priming effect, which was demonstrated in responding to related targets. In particular, the lexical decisions to targets that were preceded by primes with a large number of ANs were slower. The effects were not modulated by stimulus onset asynchrony. The results are discussed in relation to automatic and strategic mechanisms of word processing. These findings have important implications for studies in the field of word recognition.
\end{abstract}

One way to evaluate the number of associative neighbors (ANs) of a single word is by counting the different associations that come to mind upon presentation of that word. This measure has been termed \#ARSP (Chumbley \& Balota, 1984), SS (Buchanan, Westbury, \& Burgess, 2001), and $N$ (de Groot, 1989), and different ways of measurement have been proposed. The previous experiments have shown that lexical decision becomes faster as the number of neighbors increases (Buchanan et al., 2001; Chumbley \& Balota, 1984). In addition, Chumbley and Balota found a positive correlation between ANs and the time it takes to produce an appropriate association to a word. However, much less effort was allocated to investigating the effect of ANs on interword activation (e.g., priming).

Two general mechanisms have been suggested for the associative priming effect: automatic spreading of activation (ASA) (Balota \& Lorch, 1986; Collins \& Loftus, 1975) and strategic processes (see, e.g., Balota \& Lorch, 1986; de Groot, 1984). Strategic processes can be prelexical-for example, the expectancy set (Becker, 1976, 1980) — or postlexical — for example, the postlexical coherence check (de Groot, 1984). These mechanisms can be used to predict the influence of ANs on priming. According to ASA, when a concept is presented, activation spreads to neighbor concepts. Having a large number of related concepts results in each concept receiving less activation (Collins \& Loftus, 1975). Hence, in this case the prime's ANs should influence responding to related targets. Because this process is automatic, we would not expect any inhibition for unrelated targets (Neely, 1977), and therefore no influence of ANs on the response to those targets. With prelexical strategic processes, presentation of a prime should produce a set of related words, among which the participant searches for the target. If the target is part of the set, responding is facilitated. The size of the expectancy set increases with the number of the prime's neighbors. Because search time through this set correlates with set size, larger sets lead to slower responding to the target (Becker, 1976, 1980). In 1980, Becker demonstrated that it is possible to affect the composition of the expectancy set by manipulating the composition of the stimulus list. Accordingly, we hypothesized that the number of ANs would influence the priming effect, since participants would create a larger expectancy set when the prime had more neighbors. Larger sets would then lead to slower responses to related targets. In addition, because of the same mechanisms, the size of the expectancy set should also affect unrelated targets (Becker, 1980). Another strategic process, the postlexical check-an examination of the relation of the target to the prime - might predict similar results when target words differ in ANs. That is, targets with many ANs would have a weaker connection to the prime, and therefore slower responding than would targets with few ANs.

Cañas and Bajo (1994) found that participants responded more slowly to related targets preceded by primes with many ANs rather than primes with few ANs. Cañas and Bajo attributed these findings to a prelexical strategy in which participants had to search a larger expectancy set for targets preceded by a prime with many ANs. We attempted to replicate these results using Hebrew word norms collected in a discrete word association task ${ }^{1}(\mathrm{Ru}-$ binsten, Anaki, Henik, Drori, \& Faran, 2005) and found an unexpected correlation between the pattern of results and the academic characteristics of participants: ANs did

I. Ron-Kaplan, inbalro@bgu.ac.il 
not affect priming in university students, whereas they did modulate priming in community college students. In Israel, acceptance to a university requires, on average, higher achievement than acceptance to a community college. ${ }^{2}$ Therefore, we suspected that this pattern of results might be attributed to differences in verbal ability. This pattern is consistent with the compensatory hypothesis presented by Stanovich (1980) regarding a difference in the use of context by poor versus good readers. He suggested that poor readers compensate for their poor ability to recognize single words by using context. Their slower recognition thus allows them to rely more on contextual factors.

Gernsbacher and colleagues (Gernsbacher \& Faust, 1991; Gernsbacher, Varner, \& Faust, 1990) showed that lower reading comprehension results in difficulties with suppressing irrelevant information, such as the irrelevant meaning of a word. Those with higher comprehension skills also suffer from irrelevant interference, but such interference disappears if the probe does not appear immediately after the sentence. Priming studies found a positive correlation between verbal ability and semantic priming (Larkin, Woltz, Reynolds, \& Clark, 1996; Woltz, 1990).

We examined whether individual differences modulated the relationship between ANs and priming. Pilot studies and the previous findings described above led us to hypothesize that the effects of ANs on priming are contingent on verbal abilities. We hypothesized that students with low verbal ability (LVA) would rely more on a word's neighbors. Hence, ANs should modulate priming in LVA students but not in students with high verbal ability (HVA). We expected these differences to emerge specifically from differences in related trials, which should be influenced by number of ANs in the LVA group but not in the HVA group.

These differences might be the result of different patterns of spreading activation or an outcome of the different strategic mechanisms adopted by the students (e.g., search of an expectancy set). What follows includes an exploratory study with a long stimulus onset asynchrony (SOA) and a replication with both long and short SOAs. Our participants were college students characterized by heterogeneity in verbal ability.

\section{EXPERIMENT 1}

\section{Method}

Participants. One hundred and fifty-three students from Sapir College were given a 40-item Hebrew verbal ability test (Fischman, 1982). Participant scores ranged from 15 to 38, with a mean score of 27.13 (SD 5.24). The lowest $10 \%$ of this sample (18 students; 3 males) constituted the LVA group, and the top 10\% (18 students; 10 males) were the HVA group. All participants received course credit for their participation, had normal or corrected-to-normal vision, were native Hebrew speakers, and had no diagnosed learning disabilities.

Stimuli. Forty pairs of related critical words were selected from the Hebrew association norms (Rubinsten et al., 2005; see the Appendix for the critical stimuli). Of these stimuli, 20 pairs included primes with many ANs (mean 34.15, range 31-43), and 20 included primes with few ANs (mean 15.7, range 12-18). Each target was the primary associative response for its prime. The primes were equated on various attributes (e.g., word length, concreteness), and targets were equated on word length (see Table 1 ). In addition to the 40 related pairs, there were 40 unrelated pairs (formed by re-pairing the primes and targets of the related pairs) and 40 word-nonword pairs, 20 for the primes with many ANs and 20 for the primes with few ANs. The nonword targets were created by recombining letters of valid Hebrew words and forming meaningless but pronounceable letter strings.

Each participant was tested in four blocks of 80 trials each. A block was composed of 20 related pairs, 20 unrelated pairs, and 40 word-nonword pairs. The total number of trials per participant was 320. Each target in the word-word trials was presented four times across the experiment, once in each block (twice as a related target and twice as an unrelated target). Each target in the word-nonword pairs was also presented four times, once in each block. A set of 16 practice trials was created, containing the same proportion of trials for each condition as in the experimental blocks. The results of practice trials were not included in the analysis. Practice and experimental trials were presented in different random orders for each participant.

Procedure. Participants were tested individually, each seated approximately $50 \mathrm{~cm}$ from a computer screen. The experiment was conducted on an IBM R-40 laptop computer with a 15-in. screen, controlled by E-Prime software.

Each trial began with a central fixation asterisk for $250 \mathrm{msec}$, followed by a 250 -msec blank interval and then a 150 -msec central prime. After $850 \mathrm{msec}$ a central target appeared, and it remained in view until a response was recorded, but not longer than 3,000 msec. The next trial began 1,000 msec following the participant's response. Participants were asked to respond as quickly and accurately as possible by pressing the "L" key with the right index finger for word and the "A" key with the left index finger for nonword. Reaction time (RT) was measured in milliseconds from target onset until the participant's keypress. A mistake was followed by a short tone.

\section{Results}

Error trials and extreme RTs (shorter than 200 msec or longer than 2,000 msec) were excluded from the analysis $(0.3 \%$ of all trials). Errors were analyzed and showed no effects for either words or nonwords.

For each participant in each condition, mean RTs for word stimuli were computed. Mean RTs were subjected to a four-way ANOVA, with verbal ability group (HVA, LVA) as a between-subjects factor and relatedness (related, unrelated), ANs (few, many), and block (1-4) as within-subjects factors. Mean RTs as a function of condi-

Table 1

Mean Values for Different Attributes of the Two Word Lists (Few and Many Associative Neighbors [ANs])

\begin{tabular}{lrrrrr}
\hline & \multicolumn{2}{c}{ Few ANs } & & \multicolumn{2}{c}{ Many ANs } \\
\cline { 2 - 3 } \cline { 6 - 6 } & $M$ & $S E$ & & $M$ & \multicolumn{1}{c}{$S E$} \\
\hline Prime words & & & & & \\
$\quad$ Concreteness (1-7 scale) & 5.35 & 1.27 & & 5.08 & 1.63 \\
$\quad$ Familiarity (1-7 scale) & 5.95 & 0.45 & & 5.91 & 0.75 \\
$\quad$ Number of letters & 4.30 & 0.86 & & 4.85 & 1.01 \\
$\quad$ Orthography & 20.65 & 12.70 & & 20.20 & 12.00 \\
$\quad$ Proportion & 25.25 & 2.31 & & 25.30 & 2.45 \\
Target words & & & & & \\
$\quad$ Number of letters & 3.60 & 0.88 & & 3.90 & 1.13 \\
\hline
\end{tabular}

Note-There were no significant differences between the lists for the attributes above. 
Table 2

\begin{tabular}{|c|c|c|c|c|c|c|c|c|}
\hline & \multicolumn{4}{|c|}{ Low Verbal Ability } & \multicolumn{4}{|c|}{ High Verbal Ability } \\
\hline & \multicolumn{2}{|c|}{ Few ANs } & \multicolumn{2}{|c|}{ Many ANs } & \multicolumn{2}{|c|}{ Few ANs } & \multicolumn{2}{|c|}{ Many ANs } \\
\hline & $M$ & $S E$ & $M$ & $S E$ & $M$ & $S E$ & $M$ & $S E$ \\
\hline \multicolumn{9}{|c|}{ Words } \\
\hline RT & & & & & & & & \\
\hline Relate & 635 & 36 & 666 & 39 & 534 & 36 & 543 & 39 \\
\hline Unrelated & 667 & 40 & 655 & 38 & 560 & 40 & 558 & 38 \\
\hline Priming effect & 32 & & -11 & & 26 & & 15 & \\
\hline \multicolumn{9}{|l|}{ Percentage of errors } \\
\hline Related & 1.1 & & 2.5 & & 1.7 & & 1.7 & \\
\hline Unrelated & 1.4 & & 2.1 & & 2.1 & & 2.5 & \\
\hline \multicolumn{9}{|c|}{ Nonwords } \\
\hline RT & 736 & 41 & 722 & 43 & 609 & 41 & 602 & 43 \\
\hline Percentage of errors & 3.9 & & 2.4 & & 2.6 & & 2.4 & \\
\hline
\end{tabular}

tion are presented in Table 2. Nonwords were subjected to a similar ANOVA without the relatedness factor.

Words. The ANOVAs yielded a significant threeway interaction between group, ANs, and relatedness $\left[F(1,34)=4.300, M S_{\mathrm{e}}=2,241, p<.05\right]$. This interaction was the result of a difference between the simple interactions of ANs and relatedness in the two groups. In the HVA group, priming was the same, regardless of ANs $[F(1,17)<1$, n.s.]. In contrast, ANs and relatedness interacted significantly in the LVA group $[F(1,17)=13.241$, $\left.M S_{\mathrm{e}}=2,491, p<.01\right]$. Here, priming was evident for targets with few-AN primes $\left[F(1,17)=12.432, M S_{\mathrm{e}}=\right.$ $2,936, p<.01]$ and absent for targets with many-AN primes $\left[F(1,17)=1.989, M S_{\mathrm{e}}=2,173\right.$, n.s. $]$. Because our hypothesis was specifically directed toward examining differences in related trials, we further tested the simple effects in the LVA group. For related trials, responding was faster to targets with few-AN primes than to targets with many-AN primes $\left[F(1,17)=14.604, M S_{\mathrm{e}}=2,300\right.$, $p<.001]$. For unrelated trials, the prime's ANs did not affect performance $\left[F(1,17)=1.893, M S_{\mathrm{e}}=2,855\right.$, n.s. $]$. Although the effect of block was significant $[F(3,102)=$ 14.997, $\left.M S_{\mathrm{e}}=8,850, p<.001\right]$ because of decreased RTs as the blocks advanced, block did not interact with any of the other variables.

Nonwords. Three main effects were significant for nonwords: group $\left[F(1,34)=17.526, M S_{\mathrm{e}}=62,654\right.$, $p<.001$ ], because of faster RTs in the HVA group; ANs $\left[F(1,34)=5.574, M S_{\mathrm{e}}=1,464, p<.05\right]$, because of faster RTs for targets with many-AN primes; and block $\left[F(3,102)=32.914, M S_{\mathrm{e}}=4,909, p<.001\right]$, because of decreased RTs as the blocks advanced.

\section{Discussion}

We examined whether verbal ability modulates the influence of ANs on word processing and found that verbal ability does indeed modulate the AN effect on priming. Specifically, for the LVA group there was a significant interaction between ANs and relatedness, which was the result of an AN effect in related but not in unrelated trials. In the HVA group, there was a significant priming effect, regardless of ANs. The results obtained for the LVA group replicated previous results (Cañas \& Bajo, 1994).

Assuming that the pairs of words used in the experiment differed in prime ANs and not in target ANs, ${ }^{3}$ the modulation of priming by ANs in the LVA group can be explained by one or both of two mechanisms: ASA (Collins \& Loftus, 1975) and expectancy sets (Becker, 1976, 1980). In the case of ASA, different amounts of activation would spread to related words in the case of primes with many or with few ANs. When a prime had many ANs, each concept would get less activation than when the prime had few ANs. In the case of expectancy sets, participants presented with a many-AN prime would create a larger set than would those presented with a few-AN prime. Because it takes longer to search a larger than to search a smaller set, RTs would be longer to many-AN than to few-AN prime-target pairs. These two possibilities suggest two alternative mechanisms that might be employed by LVA participants. The fact that responses to unrelated targets were not influenced by the ANs of the preceding prime supports the ASA interpretation. This notion needs further examination.

We conducted Experiment 2, which both replicated the first experiment by featuring a long SOA and expanded on our previous paradigm by adding a short SOA. The short SOA allowed us to check whether the effect of ANs on priming is still evident in the LVA group when only automatic processes have time to occur (Neely, 1977).

\section{EXPERIMENT 2}

\section{Method}

Participants. A new pool of participants was gathered by administering a Hebrew verbal ability test (Fischman, 1982) to 311 students from Sapir and Achva colleges. Two groups of participants were selected: the LVA group (38 students; 8 males) from the lowest $10 \%$ of scores and the HVA group (37 students; 13 males) from the top $10 \%$.

Stimuli and Procedure. The stimuli and procedure were the same as in Experiment 1, except in the following respects. (1) The list of stimuli was larger, this time including 26 pairs of items in each condition (related, unrelated, and nonwords). (2) Participants 
were tested in two blocks only. Each target in the word-word trials was presented a total of twice, in one block as part of a related pair and in the other as part of an unrelated pair. Each target in the word-nonword pairs was also presented twice, once in each block. (3) Participants from each of the two groups were assigned randomly to an SOA condition. The long-SOA condition had the same SOA as in Experiment 1. In the short-SOA condition, the target appeared $50 \mathrm{msec}$ after the disappearance of the prime.

\section{Results}

Error trials and extreme RTs were excluded from the analysis $(0.2 \%$ of all trials). Two of the participants were excluded from the analysis because of large error rates $(>10 \%)$. For each participant in each condition, mean RTs were computed and subjected to a five-way ANOVA, with verbal ability group and SOA (short, long) as betweensubjects factors, and relatedness, ANs, and block $(1,2)$ as within-subjects factors. Nonwords were analyzed in a four-way analysis, without the relatedness factor. Mean RTs as a function of condition are presented in Tables $3 \mathrm{~A}$ and 3B (for the LVA and HVA groups, respectively).

Words. The three-way interaction between group, ANs, and relatedness was significant $\left[F(1,69)=9.326, M S_{\mathrm{e}}=\right.$ $1,646, p<.01]$ and was a result of the different patterns of interaction between ANs and relatedness for the two groups. The HVA group showed no interaction between those two factors $\left[F(1,34)=1.619, M S_{\mathrm{e}}=1,109\right.$, n.s. $]$, whereas the LVA group showed a significant interaction $\left[F(1,35)=8.224, M S_{\mathrm{e}}=2,168, p<.01\right]$. For the LVA participants, priming was evident in targets with fewAN primes $\left[F(1,35)=20.344, M S_{\mathrm{e}}=1,425, p<.001\right]$ and absent for targets with many-AN primes $(F<1)$. As in Experiment 1, the simple effects of ANs on relatedness were examined for the LVA group. For related trials, responses to targets with few-AN primes were faster than those to targets with many-AN primes $[F(1,35)=$ $\left.25.279, M S_{\mathrm{e}}=2,275, p<.001\right]$, whereas prime ANs did not modulate responding in unrelated trials $[F(1,35)=$ $1.406, M S_{\mathrm{e}}=1,847$, n.s.].

As in Experiment 1, the LVA and HVA groups differed in general RTs. One can speculate that the pattern of in- teraction between ANs and relatedness in the LVA group was the result of their general slowness, which allowed the effect of ANs to emerge. In accordance with the recommendation of Faust, Balota, Spieler, and Ferraro (1999), we preformed an ANOVA after transforming the raw data into $z$ scores. For each participant, the mean of each condition was subtracted from the participant overall mean and divided by the standard deviation for the same condition. As in the results reported above, the three-way interaction between group, ANs, and relatedness was significant $\left[F(1,69)=6.298, M S_{\mathrm{e}}=0.0514, p<.01\right]$. The HVA group showed no interaction between ANs and relatedness $(F<1)$. In contrast, the LVA group showed a significant interaction $\left[F(1,35)=9.257, M S_{\mathrm{e}}=0.0548, p<.01\right]$; the simple effect of ANs for them was significant for related trials $\left[F(1,35)=31.024, M S_{\mathrm{e}}=0.0537, p<.001\right]$, with faster responses for targets with few-AN primes, but there was no AN effect for unrelated trials.

Nonwords. Three main effects were significant: group $\left[F(1,69)=17.974, M S_{\mathrm{e}}=40,156, p<.001\right]$, because of faster RTs in the HVA group; ANs $[F(1,69)=15.367$, $\left.M S_{\mathrm{e}}=1,014, p<.001\right]$, because of faster RTs for targets with many-AN primes; and block $[F(3,69)=21.394$, $\left.M S_{\mathrm{e}}=4,059, p<.001\right]$, because of faster responses in the second block.

Errors. For words, there were three significant effects: ANs $\left[F(1,69)=17.368, M S_{\mathrm{e}}=0.339, p<.001\right]$, with fewer errors for targets with few-AN primes; relatedness $\left[F(1,69)=10.583, M S_{\mathrm{e}}=0.239, p<.01\right]$, with more errors in response to unrelated targets; and block $[F(1,69)=$ $\left.11.303, M S_{\mathrm{e}}=0.202, p<.01\right]$, with more errors in the first block. For nonwords, there was a significant effect only for ANs $\left[F(1,69)=9.547, M S_{\mathrm{e}}=0.976, p<.01\right]$, with more errors for targets with few-AN primes. These error effects were similar to the RT effects, and therefore cannot be explained by a speed-accuracy trade-off.

\section{Discussion}

Experiment 2 aimed to investigate the effect of ANs and verbal ability on priming, using both a short and a

Table 3A

Mean Reaction Times (RTs, in Milliseconds) and Percentages of Errors as Functions of Type, Stimulus Onset Asynchrony (SOA), Number of Associative Neighbors (ANs), and Relatedness for Low-Verbal-Ability Participants

\begin{tabular}{|c|c|c|c|c|c|c|c|c|}
\hline & \multicolumn{4}{|c|}{ Short SOA } & \multicolumn{4}{|c|}{ Long SOA } \\
\hline & \multicolumn{2}{|c|}{ Few ANs } & \multicolumn{2}{|c|}{ Many ANs } & \multicolumn{2}{|c|}{ Few ANs } & \multicolumn{2}{|c|}{ Many ANs } \\
\hline & $M$ & $S E$ & $M$ & $S E$ & $M$ & $S E$ & $M$ & $S E$ \\
\hline \multicolumn{9}{|c|}{ Words } \\
\hline \multicolumn{9}{|l|}{ RT } \\
\hline Related & 615 & 30 & 648 & 31 & 574 & 31 & 620 & 31 \\
\hline Unrelated & 638 & 30 & 638 & 30 & 607 & 30 & 624 & 31 \\
\hline Priming effect & 23 & & -10 & & 33 & & 4 & \\
\hline \multicolumn{9}{|l|}{ Percentage of errors } \\
\hline Related & 1.8 & & 2.8 & & 1.3 & & 2.4 & \\
\hline Unrelated & 1.8 & & 5.5 & & 2.1 & & 4.5 & \\
\hline \multicolumn{9}{|c|}{ Nonwords } \\
\hline RT & 739 & 33 & 721 & 33 & 743 & 34 & 728 & 34 \\
\hline Percentage of errors & 5.9 & & 3.2 & & 4.8 & & 3.4 & \\
\hline
\end{tabular}


Table 3B

Mean Reaction Times (RTs, in Milliseconds) and Percentages of

Errors as Functions of Type, Stimulus Onset Asynchrony (SOA), Number of Associative Neighbors (ANs), and Relatedness for High-Verbal-Ability Participants

\begin{tabular}{|c|c|c|c|c|c|c|c|c|}
\hline & \multicolumn{4}{|c|}{ Short SOA } & \multicolumn{4}{|c|}{ Long SOA } \\
\hline & \multicolumn{2}{|c|}{ Few ANs } & \multicolumn{2}{|c|}{ Many ANs } & \multicolumn{2}{|c|}{ Few ANs } & \multicolumn{2}{|c|}{ Many ANs } \\
\hline & $M$ & $S E$ & $M$ & $S E$ & $M$ & $\overline{S E}$ & $M$ & $S E$ \\
\hline \multicolumn{9}{|c|}{ Words } \\
\hline \multicolumn{9}{|c|}{ RT } \\
\hline Related & 571 & 32 & 573 & 32 & 538 & 30 & 543 & 31 \\
\hline Unrelated & 587 & 31 & 595 & 32 & 559 & 30 & 578 & 30 \\
\hline Priming effect & 16 & & 22 & & 21 & & 35 & \\
\hline \multicolumn{9}{|l|}{ Percentage of errors } \\
\hline Related & 0.7 & & 3.4 & & 1.4 & & 2.4 & \\
\hline Unrelated & 2.5 & & 2.3 & & 2.4 & & 3.2 & \\
\hline \multicolumn{9}{|c|}{ Nonwords } \\
\hline RT & 651 & 35 & 630 & 34 & 628 & 33 & 624 & 33 \\
\hline Percentage of errors & 4.9 & & 3.3 & & 3.4 & & 3.5 & \\
\hline
\end{tabular}

long SOA. The results replicated those of Experiment 1 and showed no influence of SOA on the pattern of results. For both SOAs, LVA participants showed an interaction between ANs and priming in which responding to related targets was faster when they were preceded by few-AN primes rather than many-AN primes. As in Experiment 1, unrelated targets were not influenced by the prime's ANs. HVA participants showed significant priming that was not modulated by the prime's ANs.

\section{GENERAL DISCUSSION}

We found different patterns of results for the two verbal ability groups: Prime ANs modulated the priming effect in LVA but not in HVA participants, and this difference in the patterns of results cannot be explained by general differences in RTs. Although the LVA group was slower to respond than the HVA group (on average, 113 and $69 \mathrm{msec}$ slower in Experiments 1 and 2, respectively), data transformation to standard scores revealed the same pattern of results. It seems that the two groups use associative neighbors differently in a lexical decision task. Following Stanovich's (1980) suggestion that poor readers compensate for their difficulties by using context more than good readers do, we believe that LVA students use ANs to compensate for their difficulties in producing lexical decisions.

The influence of ANs on priming in the LVA group was evident both with short and long SOAs. Cañas and Bajo (1994) used a long SOA and explained similar findings by suggesting that their participants used a prelexical strategic process - that is, that they used the prime to create an expectancy set that was searched to facilitate the response to the target. The present results raise the possibility that this effect might not necessarily be strategic, since the effect was found with a short SOA as well, thus favoring the ASA mechanism (i.e., responding to related targets was slower with many-AN primes than with few-AN primes because each association received less activation). Note that we cannot rule out the possibility that participants used a prelexical strategic process in the long-SOA condition. To differentiate between the two possibilities (ASA and expectancy set), one could manipulate the proportion of related pairs (Neely, Keefe, \& Ross, 1989) in longSOA conditions and check whether the AN modulation of priming increases when the use of an expectancy set is encouraged. The possibility that a postlexical mechanism is responsible for AN modulation of priming in the LVA group seems less reasonable, because it would require that the two stimulus lists (many- and few-AN primes) have different numbers of target ANs. ${ }^{4}$

HVA participants showed a priming effect that was not modulated by prime ANs and was the same regardless of SOA. This priming effect could be the result of other processes - for example, of a postlexical coherence check, which would facilitate responses to related targets but would not produce any additional advantage for targets with few-AN primes.

We do not suggest that HVA participants have a different semantic net or that their net activation somehow spreads differently. In another experiment conducted in our laboratory, we found that the generation of associations was positively correlated with ANs for both HVA and LVA groups - that is, responding was slower to words with many ANs (see also Chumbley \& Balota, 1984).

We believe that because LVA participants have to compensate for their poor verbal abilities, they learn to use word associations more when they have to process a word. This use is not necessarily strategic or attended, and it might result from the way they refer to concepts, by relying on their associations. Such processes would be similar to the use of different routes for processing words by different populations of readers in Coltheart, Rastle, Perry, Langdon, and Ziegler's (2001) dual route cascade (DRC) model. We suggest that participants with low verbal ability rely more on indirect routes, such as the DRC lexicalsemantic route, when having to process a concept. Within this framework, HVA participants would commonly use the 
direct route rather than the lexical-semantic one, although they can use the lexical-semantic route when necessary.

Our findings show that the number of associative neighbors of a word can influence not only word processing but also the processing of its neighbors. This influence is modulated by verbal ability. That is, responding to target words is influenced by prime ANs only in people with low verbal ability. These findings have important implications for studies in the field of language processing, since modeling and studies of various processes should take into account the influence of participant characteristics.

\section{AUTHOR NOTE}

The authors thank Desiree Meloul for her suggestions and helpful corrections on earlier versions of the manuscript. Correspondence relating to this article may be sent to I. Ron-Kaplan, Department of Behavioral Sciences, Ben-Gurion University of the Negev, Beer-Sheva, Israel (e-mail: inbalro@bgu.ac.il).

\section{REFERENCES}

Balota, D. A., \& LoRCH, R. F. (1986). Depth of automatic spreading activation: Mediated priming effects in pronunciation but not in lexical decision. Journal of Experimental Psychology: Learning, Memory, \& Cognition, 12, 336-345.

BECKER, C. A. (1976). Allocation of attention during visual word recognition. Journal of Experimental Psychology: Human Perception \& Performance, 2, 556-566.

Becker, C. A. (1980). Semantic context effects in visual word recognition: An analysis of semantic strategies. Memory \& Cognition, $\mathbf{8}$, 493-512.

Buchanan, L., Westbury, C., \& Burgess, C. (2001). Characterizing semantic space: Neighborhood effects in word recognition. Psychonomic Bulletin \& Review, 8, 531-544.

CAÑas, J. J., \& BaJo, M. T. (1994). Strategic associative priming in the lexical decision task. Quarterly Journal of Experimental Psychology, 47A, 383-405.

Chumbley, J. I., \& Balota, D. A. (1984). A word's meaning affects the decision in lexical decision. Memory \& Cognition, 12, 590-606.

Collins, A. M., \& LofTus, E. F. (1975). A spreading-activation theory of semantic processing. Psychological Review, 82, 407-428.

Coltheart, M., Rastle, K., Perry, C., Langdon, R., \& Ziegler, J. (2001). DRC: A dual route cascaded model of visual word recognition and reading aloud. Psychological Review, 108, 204-256.

DE Groot, A. M. B. (1984). Primed lexical decision: Combined effects of the proportion of related prime-target pairs and the stimulus-onset asynchrony of prime and target. Quarterly Journal of Experimental Psychology, 36A, 253-280.

DE GROOT, A. M. B. (1989). Representational aspects of word imageability and word frequency as assessed through word association. Journal of Experimental Psychology: Learning, Memory, \& Cognition, 15, 824-845.

Faust, M. E., Balota, D. A., Spieler, D. H., \& Ferraro, F. R. (1999). Individual differences in information-processing rate and amount: Implications for group differences in response latency. Psychological Bulletin, 125, 777-799.

Fischman, E. (1982). [Vocabulary subtest from the Intellectual Differential Aptitude Test (IDAT) test battery (in Hebrew)]. Center for Technological Education, Holon, Israel.

Gernsbacher, M. A., \& FAUST, M. E. (1991). The mechanism of suppres- sion: A component of general comprehension skill. Journal of Experimental Psychology: Learning, Memory, \& Cognition, 17, 245-262.

Gernsbacher, M. A., Varner, K. R., \& Faust, M. E. (1990). Investigating differences in general comprehension skill. Journal of Experimental Psychology: Learning, Memory, \& Cognition, 16, 430-445.

Larkin, A. A., Woltz, D. J., Reynolds, R. E., \& Clark, E. (1996). Conceptual priming differences and reading ability. Contemporary Educational Psychology, 21, 279-303.

Neely, J. H. (1977). Semantic priming and retrieval from lexical memory: Roles of inhibitionless spreading activation and limitedcapacity attention. Journal of Experimental Psychology: General, 106, 226-254.

Neely, J. H., Keefe, D. E., \& Ross, K. L. (1989). Semantic priming in the lexical decision task: Roles of prospective prime-generated expectancies and retrospective semantic matching. Journal of Experimental Psychology: Learning, Memory, \& Cognition, 15, 1003-1019.

Rubinsten, O., Anaki, D., Henik, A., Drori, S., \& Faran, Y. (2005). Free association norms in the Hebrew language. In A. Henik, O. Rubinsten, \& D. Anaki (Eds.), Word norms in Hebrew (pp. 17-34). BeerSheva, Israel: Ben-Gurion University of the Negev.

Stanovich, K. E. (1980). Toward an interactive-compensatory model of individual differences in the development of reading fluency. Reading Research Quarterly, 16, 32-71.

Woltz, D. J. (1990). Repetition of semantic comparisons: Temporary and persistent priming effects. Journal of Experimental Psychology: Learning, Memory, \& Cognition, 16, 392-403.

\section{NOTES}

1. The Hebrew discrete word association norms were administered as follows: Words were presented on a computer screen, and participants were asked to respond as quickly as possible with the first word that came to mind. A total of 204 participants responded to 400 words each from a data set of 800 Hebrew words. Number of ANs was the number of responses given to a single word by 102 of the participants (i.e., by half of the participant pool).

2. In Israel, entrance to institutions of higher learning is based both on matriculation and on psychometric entrance test scores. The latter are the Israeli equivalent of the SAT and cover three areas: verbal reasoning, quantitative reasoning, and English.

3. The pairs of words used in the experiments differed in prime ANs, but they were not controlled for target ANs because of limitation of the set of norms we used. One reviewer suggested that the reported effect might be due to a difference in the number of target ANs rather than to the reported difference in number of prime ANs, which might have happened if the two ANs (prime and target) were correlated. Target ANs could influence priming through a backward mechanism, such as a postlexical coherence check. In order to rule out this possibility, the Hebrew norms were searched for pairs for which we had data on both prime and target ANs. There were 114 pairs (out of 800 ) that fit this criterion, and among them no correlation was found between the ANs of the prime and of the target $(r=-.021, p=.826)$. Moreover, the predicted primeAN effect would be canceled if the two lists (with many- and few-AN primes) had different numbers of target ANs and the prime and target ANs were correlated. In that case, responses to targets that followed many-AN primes would be slower than responses to targets that followed few-AN primes. However, responses to targets with many ANs would in themselves be faster than responses to targets with few ANs (see, e.g., Buchanan et al., 2001). Hence, the existence of a prime-AN effect in the present experiment ruled out the possibility that the two stimulus lists had different mean numbers of target ANs.

4. Refer to the arguments in note 3 against systematic differences in target ANs in our experiments. 
APPENDIX

Critical Word Stimuli

\begin{tabular}{|c|c|c|c|}
\hline \multicolumn{2}{|c|}{ Many Associative Neighbors } & \multicolumn{2}{|c|}{ Few Associative Neighbors } \\
\hline Prime & Target & Prime & Target \\
\hline panther & tiger & chick & yellow \\
\hline imaginary & dream & bee & honey \\
\hline requirement & request & lighted & fire \\
\hline stopped & enough & washed & water \\
\hline gymnastic & sport & olive & green \\
\hline chameleon & colors & tail & horse \\
\hline butter & bread & hamsin (heat spell) & hot \\
\hline drawer & table & fried & chips \\
\hline justified & right & sweet & sour \\
\hline kitchen & food & sheet (bed) & bed \\
\hline kerchief & head & pot & cover \\
\hline glasses & vision & storm & rain \\
\hline kiss & love & autumn & winter \\
\hline spider & web & bell & ring \\
\hline bowl & soup & noon & morning \\
\hline radio & music & kettle & electric \\
\hline wicked & bad & clock & time \\
\hline root & tree & heat wave & heat \\
\hline dress & red & jewel & ornament \\
\hline catcher & ball & ceiling & high \\
\hline enemy & besiege & grapevine & grapes \\
\hline gown & king & fast (not eating) & kipurim \\
\hline peek & saw & beggar & poor \\
\hline monk & monastery & baked & cake \\
\hline paratrooper & red & pencil & pen \\
\hline worm & silk & expensive & money \\
\hline
\end{tabular}

(Manuscript received June 15, 2005;

revision accepted for publication May 22, 2006.) 